\title{
Projeto de Controladores Robustos Ho Usando LMIs para Controle de um Motor de Indução Trifásico com Incertezas
}

\author{
Tiago Veronese Ortunho ${ }^{1}$ \\ Instituto Federal de São Paulo, IFSP, Presidente Epitácio, SP \\ Jean Marcos de Souza Ribeiro ${ }^{2}$ \\ Departamento de Engenharia Elétrica, Unesp, Ilha Solteira, SP \\ Marcelo Carvalho Minhoto Teixeira \\ Departamento de Engenharia Elétrica, Unesp, Ilha Solteira, SP \\ José Paulo Fernandes Garcia ${ }^{4}$ \\ Departamento de Engenharia Elétrica, Unesp, Ilha Solteira, SP
}

\begin{abstract}
Resumo. O trabalho apresenta uma implementação da teoria de controladores robustos $\mathcal{H} \infty$, juntamente com a restrição de $\mathcal{D}$-Estabilidade e realimentação da integral do erro de medida usando Inequações Matriciais Lineares (LMI), considerando incertezas e objetivando minimizar distúrbio no acionamento de motor de indução trifásico (MIT). A teoria foi implementada no projeto de controle de velocidade do MIT, sendo considerados três casos distintos no controle de velocidade: i) sem incerteza, ii) incerteza na constante de tempo do rotor e, por fim, iii) incerteza na constante de tempo do rotor e do estator. Foram obtidos ótimos resultados com aplicação de carga nominal, em degrau, na ponta do eix o do MIT.
\end{abstract}

Palavras-chave. Controlador robusto $\mathcal{H} \infty$, D-Estabilidade, LMI, MIT

\section{Introdução}

Em 1972, Blaschke apresentou o princípio de controle por orientação de campo do motor de indução trifásico (MIT), porém, a dificuldade era implementá-las uma vez que a técnica de orientação de campo previa cálculos complexos, como conversão de sistemas de coordenadas móveis e manuseio das equações do modelo matemático, além de depender dos parâmetros do motor que variam de acordo com a operação. Foi proposto o desacoplamento das correntes de torque eletromagnético e de campo, possibilitando controlá-los de forma semelhante ao motor de corrente contínua. A técnica baseia-se na representação das equações eletromagnéticas da máquina em um

\footnotetext{
1 tiago.veronese@ifsp.edu.br

2 jean@dee.feis.unesp.br

3 marcelo@dee.feis.unesp.br

${ }^{4}$ jpaulo@dee.feis.unesp.br
} 
sistema de coordenada direto e quadratura (dq), com eixo direto alinhado com o vetor de fluxo. O controle é efetuado mediante variações da corrente $i_{d}$, responsável pelo campo, e corrente $i_{q}$, responsável pelo conjugado, análogo ao motor de corrente contínua [1].

Porém, é necessário que o controlador seja insensível às perturbações e incertezas, fornecendo respostas adequadas. Assim sendo, neste trabalho é proposta a utilização de técnica de controle robusto juntamente com Desigualdades Matriciais Lineares (LMIs), possibilitando o tratamento de incertezas e inclusão de restrições de desempenho [2].

\section{Modelo de estado do motor de indução}

A partir das equações dinâmicas do motor de indução com rotor em gaiola de esquilo e considerando o sistema na referência síncrona girante $\left(w_{S}\right)$ obtêm-se (1) a (11).

$$
\begin{gathered}
\frac{d}{d t} i_{d S}^{e}=-\left[\frac{R_{S}}{\sigma \cdot L_{S}}+\frac{R_{r}(1-\sigma)}{\sigma \cdot L_{R}}\right] \cdot i_{d S}^{e}+w_{S} \cdot i_{q S}^{e}+\frac{L_{m} \cdot R_{r}}{\sigma \cdot L_{S} L_{R}^{2}} \cdot \emptyset_{d R}^{e}+\frac{w_{R} \cdot L_{m}}{\sigma \cdot L_{S} \cdot L_{R}} \cdot \emptyset_{q R}^{e}+\frac{1}{\sigma \cdot L_{S}} \cdot V_{d S}^{e} \\
\frac{d}{d t} i_{q S}^{e}=-\left[\frac{R_{S}}{\sigma \cdot L_{S}}+\frac{R_{r}(1-\sigma)}{\sigma \cdot L_{R}}\right] \cdot i_{q S}^{e}-w_{S} \cdot i_{d s}^{e}+\frac{L_{m} \cdot R_{r}}{\sigma \cdot L_{S} \cdot L_{R}^{2}} \cdot \emptyset_{q R}^{e}-\frac{w_{R} \cdot L_{m}}{\sigma \cdot L_{S} \cdot L_{R}} \cdot \emptyset_{d R}^{e}+\frac{1}{\sigma \cdot L_{S}} \cdot V_{q S}^{e} \\
\frac{d}{d t} \emptyset_{d R}^{e}=\frac{L_{m} \cdot R_{r}}{L_{R}} \cdot i_{d s}^{e}-\frac{R_{r}}{L_{R}} \cdot \emptyset_{d R}^{e}+\left(w_{S}-w_{R}\right) \cdot \emptyset_{q R}^{e} \\
\frac{d}{d t} \emptyset_{q R}^{e}=\frac{L_{m} \cdot R_{r}}{L_{R}} \cdot i_{q S}^{e}-\frac{R_{r}}{L_{R}} \cdot \emptyset_{q R}^{e}-\left(w_{S}-w_{R}\right) \cdot \emptyset_{d R}^{e} \\
T_{e}=\frac{3 \cdot p}{2} \cdot \frac{L_{m}}{L_{R}} \cdot\left(i_{q S}^{e} \cdot \emptyset_{d R}^{e}-i_{d s}^{e} \cdot \emptyset_{q R}^{e}\right) \\
T_{e}-T_{L}=J \cdot \frac{d}{d t} w_{R}+B \cdot w_{R}
\end{gathered}
$$

sendo $i_{d s}^{e}$ e $i_{q S}^{e}$ componentes de eixo direto e em quadratura da corrente do estator; $R_{S}, R_{r}$, as resistências do estator e rotor; $L_{s}, L_{R}$ e $L_{m}$, as indutâncias do estator, rotor e mútua; $\emptyset_{d R}^{e}$ e $\emptyset_{q R}^{e}$ as componentes de eixo direto e quadratura do fluxo do rotor; $T_{e}$ e $T_{L}$ os torques eletromagnético e de carga; $w_{R}$ a velocidade do rotor; $p, J, B$ e $\sigma$, o $\mathrm{n}^{\mathrm{o}}$ de pares de polos, momento de inércia, coeficiente de atrito viscoso e coeficiente de dispersão do motor.

A velocidade de escorregamento é dada por (7) e o fluxo de componente direto do rotor, representado por (8). O torque eletromagnético é apresentado em (9) e a velocidade angular mecânica em (10). Após, define-se a corrente no eixo de quadratura dada em (11).

$$
\begin{gathered}
w_{e s c}=w_{S}-w_{R}=\frac{L_{m} \cdot R_{r}}{L_{R} \cdot \emptyset_{d R}^{e}} \cdot i_{q S}^{e} \\
\frac{d}{d t} \emptyset_{d R}^{e}+\frac{R_{r}}{L_{R}} \cdot \emptyset_{d R}^{e}=\frac{L_{m} \cdot R_{r}}{L_{R}} \cdot i_{d S}^{e} \\
T_{e}=\frac{3 \cdot p}{2} \cdot \frac{L_{m}{ }^{2}}{L_{R}} \cdot i_{d S}^{e}{ }^{*} \cdot i_{q S}^{e}=\frac{3 \cdot p}{2} \cdot \frac{L_{m}}{L_{R}} \cdot \emptyset_{d R}^{e} \cdot{ }^{*} \cdot i_{q S}^{e}=K_{t} \cdot i_{q S}^{e} \\
\frac{d}{d t} w_{R}=\frac{K_{t}}{J} \cdot i_{q S}^{e}-\frac{B}{J} \cdot w_{R}-\frac{1}{J} \cdot T_{L} \\
\frac{d}{d t} i_{q S}^{e}=-\frac{R_{S}}{\sigma \cdot L_{S}} \cdot i_{q S}^{e}-w_{S} \cdot i_{d S}^{e}-w_{S} \cdot \frac{L_{m} \cdot \emptyset_{d R}^{e}}{\sigma \cdot L_{S} \cdot L_{R}}+\frac{1}{\sigma \cdot L_{S}} \cdot V_{q S}^{e}
\end{gathered}
$$

Das expressões (1) a (11) é possível escrever o modelo conforme (12) a (14), sendo $\alpha$ as incertezas da planta. O torque de carga será considerado um distúrbio do sistema.

$$
\dot{x}(t)=A(\alpha) \cdot x(t)+B_{u}(\alpha) \cdot u(t)+B_{W}(\alpha) \cdot T_{L}(t) ; \quad y(t)=C(\alpha) x(t) ; \quad A(\alpha)=\left[\begin{array}{llll}
a_{11} & a_{12} & a_{13} & a_{14} \\
a_{21} & a_{22} & a_{23} & a_{24} \\
a_{31} & a_{32} & a_{33} & a_{34} \\
a_{41} & a_{42} & a_{43} & a_{44}
\end{array}\right]
$$




$$
\begin{aligned}
& a_{11}=-\left[\frac{R_{S}}{\sigma L_{S}}+\frac{R_{r}(1-\sigma)}{\sigma . L_{R}}\right] ; a_{12}=w_{S} ; a_{13}=\frac{L_{m} \cdot R_{r}}{\sigma . L_{S} \cdot L_{R}^{2}} ; a_{14}=0 ; a_{21}=-w_{S} ; a_{22}=-\frac{R_{S}}{\sigma L_{S}} ; a_{23}=-\frac{w_{S} L_{m}}{\sigma . L_{S} L_{R}} ; a_{24}=0 \\
& a_{31}=\frac{L_{m} \cdot R_{r}}{L_{R}} ; a_{32}=0 ; a_{33}=-\frac{R_{r}}{L_{R}} ; a_{34}=0 ; a_{41}=0 ; a_{42}=\frac{K_{t}}{J} ; a_{43}=0 ; a_{44}=-\frac{B}{J} \\
& B_{u}(\alpha)=\left[\begin{array}{cc}
\frac{1}{\sigma . L_{S}} & 0 \\
0 & \frac{1}{\sigma . L_{S}} \\
0 & 0 \\
0 & 0
\end{array}\right] ; B_{W}(\alpha)=\left[\begin{array}{c}
0 \\
0 \\
0 \\
-\frac{1}{J}
\end{array}\right] ; C(\alpha)=\left[\begin{array}{cccc}
1 & 0 & 0 & 0 \\
0 & 1 & 0 & 0
\end{array}\right] ; X(t)=\left[\begin{array}{c}
i_{d s}^{e} \\
i_{q s}^{e} \\
\oint_{d R}^{e} \\
w_{R}
\end{array}\right] ; u(t)=\left[\begin{array}{c}
V_{d S}^{e} \\
V_{q S}^{e}
\end{array}\right]
\end{aligned}
$$

Os dados do MIT estão representados na Tabela 1, os quais foram utilizados no trabalho [4]. O MIT possui potência de 0,25 HP, 4 polos, 1725 RPM, 380/220V, 60Hz.

Tabela 1: Dados do MIT.

\begin{tabular}{|l|l|l|l|}
\hline Dados do motor & Valores & Dados do motor & Valores \\
\hline Resistência do Estator $\left(R_{S}\right)$ & $29,5012 \Omega$ & Indutância do Estator $\left(L_{S}\right)$ & $0,0534 \mathrm{H}$ \\
\hline Resistência do Rotor $\left(R_{r}\right)$ & $17,8384 \Omega$ & Indutância do Rotor $\left(L_{R}\right)$ & $0,0637 \mathrm{H}$ \\
\hline Momento de Inércia $(J)$ & $0,0005 \mathrm{Kg} . \mathrm{m}^{2}$ & Indutância Mútua $\left(L_{m}\right)$ & $1,0417 \mathrm{H}$ \\
\hline Coeficiente de Atrito Viscoso $(B)$ & $0,003 \mathrm{~m} / \mathrm{rad} . \mathrm{s}$ & Fluxo Nominal do Rotor & $0,93 \mathrm{~Wb}$ \\
\hline
\end{tabular}

\section{Inequações Matriciais Lineares}

Uma LMI é uma desigualdade matricial do tipo $F(g)>0$, na qual $F(g): \mathrm{R}^{\mathrm{m}} \rightarrow \mathrm{R}^{\mathrm{q} \times \mathrm{q}}$ é simétrica e afim nas variáveis de busca que são representadas pelo vetor $g$. Assim ela pode ser genericamente representada na forma $F(g)=F_{0}+\sum_{i=1}^{m} g_{i} F_{i}>0$, no qual $F_{i}=F_{i}{ }^{\prime} \in \mathrm{R}^{\mathrm{q} \times \mathrm{q}}$ são matrizes constantes e $g \in \mathrm{R}^{\mathrm{m}}$ é o vetor de variáveis, com elementos escalares, a ser determinado de forma a satisfazer a desigualdade $A^{\prime} . P+P . A<0$.

\subsection{Controlador robusto $\mathcal{H} \infty$ usando LMIs}

Considerando o sistema em variáveis de estado conforme (15) e $u(t)=K \cdot x(t)$, obtémse uma nova variável definida como $A_{N}=A(\alpha)+B_{u}(\alpha)$. $K$. Com o intuito de minimizar o custo garantido $H \infty$ entre o distúrbio $w(t)$ e a saída $y(t)$, aplica-se (16), transformando este conjunto em LMI e, aplicando o complemento de Schur, obtém (17). Ao substituir a variável $A_{N}$ é obtida a restrição (18) com controlador $K=Z$. $W^{-1}$ [5].

$$
\begin{gathered}
\dot{x}(t)=A(\alpha) \cdot x(t)+B_{u}(\alpha) \cdot u(t)+B_{w}(\alpha) \cdot T_{L}(t) ; y(t)=C(\alpha) \cdot x(t)+D \\
{\left[\begin{array}{cc}
A_{N} \cdot W+W \cdot A_{N}^{\prime}+B_{W} \cdot B_{W}^{\prime} & W \cdot C^{\prime}+B \cdot D^{\prime} \\
C \cdot W+D \cdot B^{\prime} & -\mu \cdot I
\end{array}\right]<0 ; W>0} \\
{\left[\begin{array}{ccc}
A_{N} \cdot W+W \cdot A_{N}^{\prime} & W \cdot C^{\prime} & B_{W} \\
C \cdot W & -\mu \cdot I & D \\
B_{W}^{\prime} & D^{\prime} & -I
\end{array}\right]<0 ; W>0} \\
{\left[\begin{array}{ccc}
A \cdot W+B_{u} \cdot Z+W \cdot A^{\prime}+Z^{\prime} \cdot B_{u}^{\prime} & W \cdot C^{\prime}+Z^{\prime} \cdot D & B_{W} \\
C . W+D \cdot Z & -\mu \cdot I & D \\
B_{W}^{\prime} & D^{\prime} & -I
\end{array}\right]<0 ; W>0}
\end{gathered}
$$

\subsection{Controlador robusto $\mathcal{H} \infty$ com $\mathcal{D}$-Estabilidade usando LMIs}

O trabalho [6] restringe a região do controlador para uma de interesse, $S(\alpha, r, \theta)$, de números complexos $(x+j y)$ que satisfazem as restrições em 3 regiões descritas em (19).

$$
S(\alpha, r, \theta)=\{\mathrm{x}<-\alpha<0 ; \quad|\mathrm{x}+\mathrm{jy}|<r ; \quad \tan (\theta) \mathrm{x}<-|\mathrm{y}|
$$

Alocar autovalores na região $S(\alpha, r, \theta)$, permite efetuar projetos que restrinjam a 
porcentagem de overshoot, tempo de subida e tempo de estabelecimento, através de LMIs $[3,6]$. As restrições para o dimensionamento do controlador são apresentadas em (20).

$$
X>0 ; A . X+X . A^{\prime}+2 . \alpha \cdot X<0 ;\left(\begin{array}{cc}
-r . X & A . X \\
X . A^{\prime} & -r . X
\end{array}\right)<0 ;\left(\begin{array}{cc}
\sin \theta \cdot\left(A \cdot X+X \cdot A^{\prime}\right) & \cos \theta \cdot\left(A \cdot X-X . A^{\prime}\right) \\
\cos \theta \cdot\left(X \cdot A^{\prime}-A \cdot X\right) & \sin \theta \cdot\left(A \cdot X+X \cdot A^{\prime}\right)
\end{array}\right)<0
$$

\subsection{Controlador robusto $\mathcal{H} \infty$ com $\mathcal{D}$-Estabilidade e realimentação da integral do erro de saída usando LMIs}

A integral é utilizada para que o sistema em regime permanente possua erro nulo para uma entrada degrau. Para o sistema em análise, as novas variáveis de estado ficam descritas pelas matrizes estendidas (21) a (23) com lei de controle $u(t)=K \cdot x(t)-K . \varepsilon(t)$ [3].

$$
\begin{aligned}
& \frac{d}{d t} \hat{x}(t)=\hat{A}(\alpha) \cdot \hat{x}(t)+\widehat{B_{u}}(\alpha) \cdot \hat{u}(t)+\widehat{B_{w}}(\alpha) \cdot T_{L}(t) \quad ; \quad y(t)=\hat{C}(\alpha) \cdot \hat{x}(t) \\
& {\left[\begin{array}{l}
\dot{x}(t) \\
\dot{\varepsilon}(t)
\end{array}\right]=\left[\begin{array}{cc}
A(\alpha) & 0 \\
-C(\alpha) & 0
\end{array}\right] \cdot\left[\begin{array}{l}
x(t) \\
\varepsilon(t)
\end{array}\right]+\left[\begin{array}{c}
B_{u}(\alpha) \\
I
\end{array}\right] \cdot\left[\begin{array}{ll}
u(t) & \operatorname{ref}(t)
\end{array}\right]+\left[\begin{array}{c}
B_{w}(\alpha) \\
0
\end{array}\right] \cdot T_{L}(t) ; y=\left[\begin{array}{lll}
C(\alpha) & 0
\end{array}\right] \cdot\left[\begin{array}{l}
x(t) \\
\varepsilon(t)
\end{array}\right]} \\
& {[\dot{\varepsilon}(t)]=\left[\begin{array}{c}
\dot{\varepsilon}_{1}(t) \\
\dot{\varepsilon}_{2}(t)
\end{array}\right]=\left[\begin{array}{c}
\emptyset_{d R}^{e}-\emptyset_{d R}^{e} \\
W_{R}^{*}-W_{R}
\end{array}\right] ;[\varepsilon(t)]=\left[\begin{array}{c}
\int \varepsilon_{1}(t) \\
\int \varepsilon_{2}(t)
\end{array}\right]=\left[\begin{array}{c}
\int\left(\emptyset_{d R}^{e}{ }^{*}-\emptyset_{d R}^{e}\right) \\
\int\left(W_{R}^{*}-W_{R}\right)
\end{array}\right] ;[\operatorname{ref}(t)]=\left[\begin{array}{c}
\emptyset_{d R}^{e}{ }^{*} \\
W_{R}^{*}
\end{array}\right]}
\end{aligned}
$$

\section{Resultados}

Foi considerado 3 casos, todos com velocidade variando da mínima até a nominal: i) sem incerteza paramétrica, ii) incerteza na constante de tempo do rotor (5\%), e iii) incerteza nas constantes de tempo do rotor (15\%) e estator (10\%). Assim, tem-se 2,4 e 8 vértices do politopo e, por convexidade, tem-se que se uma dada condição estiver satisfeita em todos os vértices, então ela também estará satisfeita para qualquer ponto pertencente ao politopo. As Tabelas 2-4 apresentam as matrizes de controladores e as normas $\mathcal{H} \infty$ para cada caso.

\begin{tabular}{|c|c|c|c|c|c|c|}
\hline Caso & \multicolumn{4}{|c|}{ Controladores } & Normas & Índices de Desempenho \\
\hline 1 & {$\left[\begin{array}{l}121,01 \\
-3,093\end{array}\right.$} & $\begin{array}{c}3,042 \\
65,831\end{array}$ & $\begin{array}{r}-4274,62 \\
3163,893\end{array}$ & $\begin{array}{l}12,753 * 10^{-12} \\
361,322 * 10^{-9}\end{array}$ & $\begin{array}{l}268,899 \\
* 10^{-6}\end{array}$ & $\alpha=1 ; r=4000 ; \theta=0,610$ \\
\hline 2 & {$\left[\begin{array}{l}136,087 \\
-3,229\end{array}\right.$} & $\begin{array}{c}3,003 \\
79,401\end{array}$ & $\begin{array}{c}-4315,395 \\
3121,294\end{array}$ & $\begin{array}{c}-60,586 * 10^{-12} \\
280,264 * 10^{-9}\end{array}$ & $\begin{array}{l}209,153 \\
* 10^{-6}\end{array}$ & $\alpha=1 ; r=4900 ; \theta=0,61$ \\
\hline 3 & {$\left[\begin{array}{l}141,16 \\
-2,934\end{array}\right.$} & $\begin{array}{r}3,003 \\
84,899\end{array}$ & $\begin{array}{r}-4373,24 \\
3123,982\end{array}$ & $\begin{array}{l}-5,589 * 10^{-12} \\
451,834 * 10^{-9}\end{array}$ & $\begin{array}{l}209,987 \\
* 10^{-6}\end{array}$ & $\alpha=1 ; r=5800 ; \theta=0,959$ \\
\hline
\end{tabular}

Tabela 2: Valores dos Controladores Robustos $\mathcal{H} \infty$ Usando LMI.

\begin{tabular}{|c|cccc|c|}
\hline Caso & \multicolumn{3}{|c|}{ Controladores } & Normas \\
\hline 1 & {$\left[\begin{array}{cccc}182908,984 & 1757,350 & -4540,484 & 0,062 \\
-8506,537 & 460780,604 & 3148,040 & -0,013\end{array}\right]$} & 0,005406 \\
\hline 2 & {$\left[\begin{array}{cccc}295251,796 & 6404,136 & -4551,659 & -0,004 \\
-11423,352 & 538621,743 & 3131,260 & -0,016\end{array}\right]$} & 0,0001 \\
\hline 3 & {$\left[\begin{array}{cccc}531380,155 & -24118,964 & -3681,375 & -0,081 \\
22143,820 & 202092,726 & 3160,050 & -0,030\end{array}\right]$} & 0,00291 \\
\hline
\end{tabular}

Tabela 3: Valores dos Controladores Robustos $\mathcal{H} \infty$ com $\mathcal{D}$ - Estabilidade Usando LMI.

Tabela 4: Valores dos Controladores Robustos $\mathcal{H} \infty$ com $\mathcal{D}$ - Estabilidade e Realimentação da Integral do Erro de Saída.

\begin{tabular}{|c|ccccc|c|c|}
\hline Caso & Controladores & Normas & Índices de Desempenho \\
\hline 1 & {$\left[\begin{array}{cccccc}185,77 & 2,90 & -3067,73 & 53,15 * 10^{-9} & -0,79 & 0,48 \\
40,31 & 118,32 & 3963,12 & 5,75 * 10^{-6} & 12,38 & 21,66\end{array}\right]$} & $\begin{array}{c}350,759 \\
* 10^{-6}\end{array}$ & $\alpha=1 ; r=7000 ; \theta=1,483$ \\
\hline 2 & {$\left[\begin{array}{cccccc}211,39 & 2,81 & -2817,31 & -358,87 * 10^{-9} & -0,92 & -0,28 \\
40,22 & 131,22 & 4014,03 & 0,001 & 9,22 & 16,48\end{array}\right]$} & 5,651 & $\alpha=1 ; r=7900 ; \theta=1,134$ \\
\hline 3 & {$\left[\begin{array}{cccccc}204,29 & 2,70 & -3484,49 & -576,93 * 10^{-9} & -0,58 & 0,26 \\
75,42 & 130,30 & 4577,49 & -65,28 * 10^{-6} & 6,49 & 13,60\end{array}\right]$} & 1,161 & $\alpha=1 ; r=8000 ; \theta=0,959$ \\
& 0 & & &
\end{tabular}

Considerando o torque de carga como um degrau de valor nominal, no instante $(\mathrm{t}=0 \mathrm{~s})$, 
foram plotados no Matlab a resposta no tempo para os casos em análise, tendo como objetivo visualizar o comportamento do sistema para os controladores desenvolvidos.

É importante esclarecer que os resultados a seguir apenas mostram a tentativa de rejeição do distúrbio (aplicação de carga) nos sinais de saída (correntes $i_{d}$ e $i_{q}$ ), sendo eles o objetivo de controle, que possuem referência igual a zero; isso não faz sentido para o controle do MIT, contudo é importante para a investigação da robustez do controlador. Em outro momento serão estimados o fluxo e velocidade do rotor, e estes estarão sujeitos à aplicação das técnicas de controle propostas. Por falta de espaço para apresentar todos resultados, as Figuras 1-9 descrevem apenas os resultados para um vértice, para cada caso e controlador, escolhido aquele que resultou em maior amplitude de sinal de saída (correntes $i_{d}$ e $i_{q}$ ).

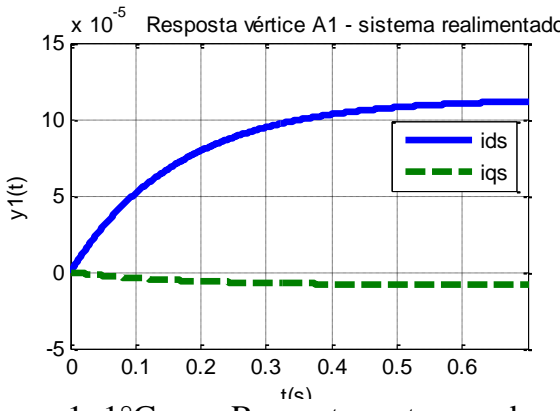

Figura $1: 1^{\circ}$ Caso - Resposta no tempo do controlador robusto $\mathcal{H} \infty$.

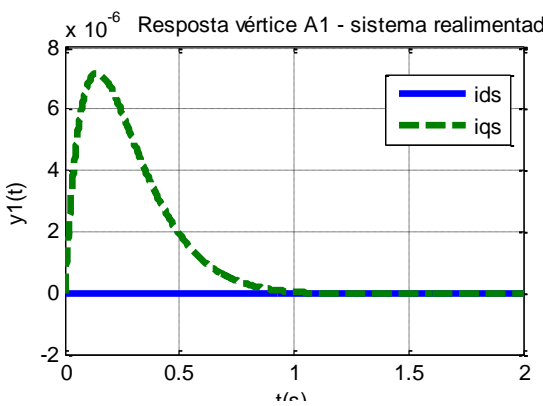

Figura 3: $1^{\circ} \mathrm{Caso}$ - Resposta no tempo do controlador robusto $\mathcal{H} \infty$ com $\mathcal{D}$ Estabilidade e integral do erro.

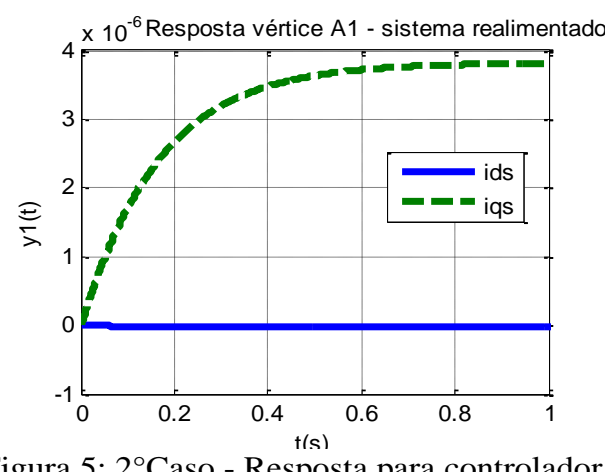

Figura 5: $2^{\circ} \mathrm{Caso}$ - Resposta para controlador $\mathcal{H} \infty$ com $\mathcal{D}$ - Estabilidade.

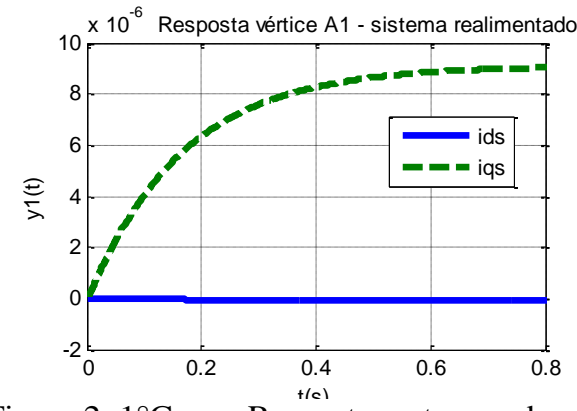

Figura 2: $1{ }^{\circ} \mathrm{Caso}$ - Resposta no tempo do controlador robusto $\mathcal{H} \infty$ com $\mathcal{D}$-Estabilidade.

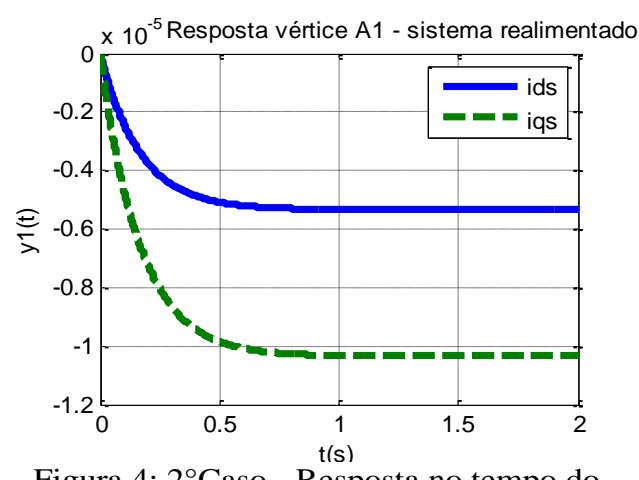

Figura 4: $2^{\circ}$ Caso - Resposta no tempo do controlador robusto $\mathcal{H} \infty$.

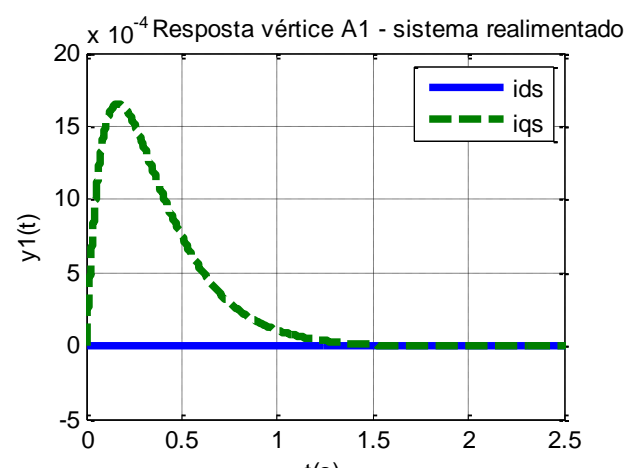

Figura 6: $2^{\circ}$ Caso - Resposta para controlador $\mathcal{H} \infty$ com $\mathcal{D}$ - Estabilidade e integral do erro. 


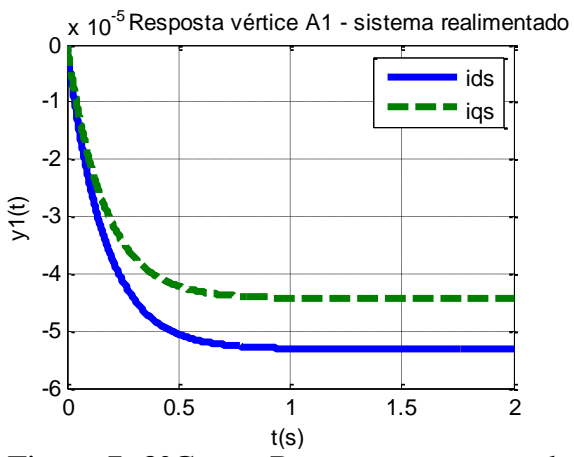

Figura 7: $3^{\circ}$ Caso - Resposta no tempo do controlador robusto $\mathcal{H} \infty$.

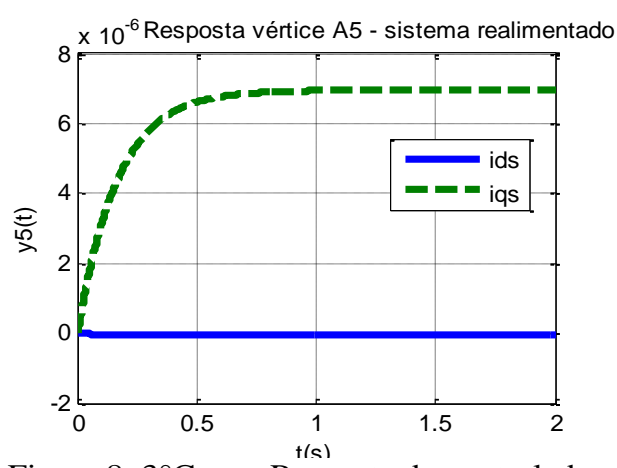

Figura 8: $3^{\circ} \mathrm{Caso}$ - Resposta do controlador

$\mathcal{H} \infty$ com $\mathcal{D}$ - Estabilidade

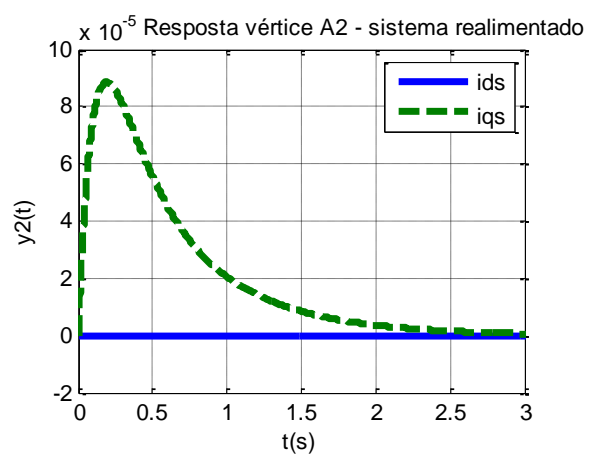

Figura 9: $3^{\circ}$ Caso - Resposta do controlador $\mathcal{H} \infty$ com $\mathcal{D}$-Estabilidade e integral do erro.

No caso 1 , o controlador $\mathcal{H} \infty$ (Figura 1) estabilizou em 0,7 seg sem oscilação, com $\mathcal{D}$ estabilidade (Figura 2) a amplitude reduziu e entrou em regime praticamente no mesmo tempo e, para o terceiro controlador (Figura 3), a saída estabiliza em 1,2 seg com pico de $8.10^{-6}$.

No segundo caso, os dois primeiros controladores entraram em regime em aproximadamente $1 \mathrm{seg}$, porém, o controlador ótimo (Figura 4) gerou uma resposta apresentando uma amplitude máxima na corrente do eixo de quadratura de aproximadamente $-1.10^{-5}$. No controlador sub-ótimo (Figura 5) a saída teve pico máximo de $4.10^{-6}$. A realimentação da integral (Figura 6) proporcionou uma resposta que estabilizou em 1,5 seg com magnitude máxima de $17 \cdot 10^{-4}$.

Por fim, no último caso o controlador ótimo (Figura 7) proporcionou resposta com máximo pico em $-5.10^{-5}$ na corrente de eixo direto, entrando em regime em aproximadamente $1 \mathrm{seg}$. Por outro lado, o controlador sub-ótimo (Figura 8) gerou uma resposta com a máxima corrente sendo a do eixo em quadratura com magnitude máxima de $6.10^{-6}$, com tempo de estabelecimento aproximadamente igual ao ótimo. O controlador com realimentação da integral (Figura 9) levou a reposta para a estabilidade no tempo máximo de 2,5 seg e com pico de aproximadamente $10 \cdot 10^{-5}$.

Os controladores geraram respostas rápidas para o sistema, ou seja, mesmo no pior caso, com muita incerteza, o tempo de estabelecimento não atingiu $3 \mathrm{seg}$, fato este muito importante para o controle de velocidade do motor de indução. Estes resultados mostram alta robustez deste controlador quando projetado utilizando a ferramenta LMI. 


\section{Conclusão}

O trabalho propiciou a análise e investigação da teoria de controle robusto $\mathcal{H} \infty$, englobando o desempenho pela restrição de $\mathcal{D}$-Estabilidade, também, o controle robusto $\mathcal{H} \infty$ com restrição de $\mathcal{D}$-Estabilidade e realimentação da integral do erro de saída, todos aplicados ao acionamento de um motor de indução trifásico. Para o projeto do controlador de velocidade foi utilizado a ferramenta LMI, considerando três casos: i) sem incertezas, ii) com incerteza na constante de tempo do rotor e, por fim, iii) incerteza nas constantes de tempo do rotor e do estator concomitantemente. Os autores estão muito otimista com os resultados obtidos com a técnica de controle robusto $\mathcal{H} \infty$ usando o critério de $\mathcal{D}$-Estabilidade e realimentação do sinal da integral do erro, pois neste caso houve uma boa rejeição ao distúrbio e estabilização do sinal de interesse em um valor com erro igual a zero.

Entende-se que este trabalho possibilitou uma ampliação da aplicação da teoria de controladores robustos $\mathcal{H} \infty$ em MITs, obtendo ótimos resultados pela análise da resposta no tempo para uma entrada degrau de carga nominal na ponta do eixo do motor.

\section{Agradecimentos}

Os autores agradecem o DEE da FE-IS/UNESP e ao IFSP/PEP por proporcionar excelentes condições de desenvolver pesquisas.

\section{Referências}

[1] B. K. Bose, Modern power electronics and ac drives, Upper Saddle River, PrenticeHall, vol. 1, (2001).

[2] S. Boyd, L. E. Ghaoui, E. Feron and V. Balakrishnan, Linear matrix inequalities in systems and control theory, SIAM studies in applied mathematics, (1994).

[3] M. V. S. Costa, V. P. Pinto, J. C. T. Campos e J. A. Nascimento, Controle por DAlocação robusta via LMI aplicado em sistemas de geração eólica, Anais do Simpósio Brasileiro de Sistemas Elétricos, (2012).

[4] A. F. A. Furtunato, A. O. Salazar e O. S. Araújo, Controlador de velocidade usando modos deslizantes suaves para um motor de indução trifásico, CONTROLE \& AUTOMAÇÃO, vol. 12, 148-155, (2001).

[5] P. Gahinet, Explicit controller formulas for LMI-based Ho synthesis, AUTOMÁTICA, vol. 32, 1007-1014, (1996).

[6] R. M. Manesco, J. H. P. Silva, M. R. Moreira, L. F. S. Buzachero, E. R. P. D. Silva, E. Assunção, M. C. M. Teixeira e R.K.H. Galvão, Estabilidade robusta $\mathrm{H} \infty$ de sistemas lineares: uma implementação em um helicóptero 3DOF de bancada, Anais do Congresso Brasileiro de Automática, (2012). 